\title{
Some studies are more equal than others
}

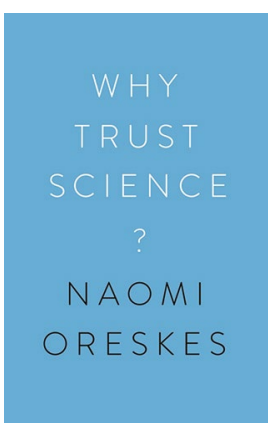

\author{
Why Trust Science? \\ By Naomi Oreskes \\ PRINCETON \\ UNIVERSITY PRESS: \\ 2019. 376PP. $€ 22$
}

\footnotetext{
$\mathrm{S}$
} ociety's trust in scientific research and its policies is under increasing attack. Although mistrust of science is not new (for instance, opposition to vaccinations has existed ever since Jenner's pioneering work), this is taking an increasingly urgent tone as the world grapples with the rapidly accelerating consequences of climate change. Mistrust of science can be woven by corporate subterfuge or individuals with a public platform to voice their opinion; regardless of motivation or qualification, anyone can express seemingly valid criticism of science and scientists alike.

Why Trust Science? by Naomi Oreskes addresses this issue by expertly discussing why science and those who dedicate their life to studying it should be the ones trusted to provide the defining conclusions on the natural world. She argues that science is not the realm of any one individual but an open society built on consensus and selfawareness whose strength is mirrored by its diversity.

Oreskes analyses influential philosophical thinking on the nature of science, its methods and how it continually subjects itself to internal scrutiny as a means to refine and improve its understanding, a process that was termed 'transformative interrogation' by the philosopher Helen Longino. Oreskes then takes four instances where science has 'gone awry' and explains how these exemplify the ability of the scientific community to inwardly reflect and reject theories that do not hold up scrutiny and achieve a broad consensus. This is concluded with a coda where she sets out five fundamental themes that she believes should govern the scientific community: consensus, method, evidence, values and humility. The subsequent four chapters are commentaries by prominent experts in the field, expanding, supporting or criticizing the arguments put forward, and to which Oreskes responds in the final chapter.

Central to Oreskes' argument is that science is reliant on consensus. True scientific progress is never based on a single experiment or individual - no matter how influential - and the scientific community functions best when it draws a range of perspectives from a diverse community. This is best demonstrated by two examples of invalidated theories: the limited energy theory (which stated that the higher education of women was pursued at the expense of their capability to reproduce) and eugenics. The latter is typically used by climate change deniers as an example of unreliable science, but Oreskes points out that these theories never had the consensus of the scientific community, being instead put forward by a homogenous group of privileged individuals who benefited from these ideas. In fact, prominent counter studies highlighting glaring inaccuracies were published at the time, but often ignored by the proponents of these theories.

She also argues that it is a fallacy to believe there is a standard (or 'textbook') scientific method that can be applied to all types of research. Science is at its weakest when it is governed by assumptions or becomes enamoured with a particular theory or method to the extent it rejects contradictory results, a trait Oreskes terms as 'methodological fetishism'. Our understanding is limited by the capability of the currently available techniques, and the onus of scientists is not to demonstrate that a theory is correct but rather that it is robust under continual scrutiny. Nothing in science is set in stone and only through experience and observation can we successfully advance our understanding.

The book comes at a pivotal moment where, despite the science behind climate change having near-universal consensus within the scientific community, counter studies from outsiders such as corporations and think-tanks - whose motivations and funding are sometimes opaque deliberately seek to muddy the waters. This follows a historical pattern of the tobacco and food industries whose survival depends on contradicting the scientific evidence that endangers their livelihood. This is further exacerbated by the media who often take scientific studies out of context, not understanding their full significance, and publish contradictory articles where, for example, foods or habits can seemingly both cure and inflict damage on our health in equal measure.

The consequence of such activities is to erode the general public's trust of science and subject scientists to a level of judgement and scepticism that we would not normally hold other professionals to. Paraphrasing Oreskes' apt words: we trust plumbers to do plumbing and nurses to do to nursing, so why can we not trust scientists to similarly do their job? These respective communities have measures to root out rogue individuals, and science is no different. The modern world is already full of examples of successful and accepted science. This demonstrates that, as a whole, society does not actually reject science. However, when confronted with "scientific claims and conclusions that clash with their economic interests or cherished beliefs", attitudes can become polarized, politicized, and open to manipulation. Oreskes hopes to demonstrate that regardless of our beliefs we share common values and goals; no one truly desires the devastating consequences of climate change.

Oreskes creates a compelling argument that demonstrates how over centuries scientists "have developed practices for identifying problems in theories and experiments". While such practices may not be infallible, collectively as a community scientists have consistently demonstrated the ability to detect errors and reconsider their views. This extensive record of experience and observation is precisely why, when a near-universal consensus over the effects of climate change has been reached by the community, we should be confident in placing our trust in the evaluation of that science and not in those who seek to undermine it from the outside. So why trust science? Because we already do.

Reviewed by Saleem Denholme Associate Editor, Communications Physics. e-mail:saleem.denholme@nature.com

Published online: 6 February 2020 https://doi.org/10.1038/s41567-020-0788-x 